\title{
Low-density lipoprotein cholesterol lowering therapy for the secondary prevention of atherosclerotic cardiovascular disease
}

${ }^{1}$ Department of Medicine, Brigham \& Women's Hospital, Harvard Medical School, Boston, MA, USA

2 Division of Cardiovascular Medicine, Brigham \& Women's Hospital, Harvard Medical School, Boston, MA, USA

*Email: rgiugliano@bwh.harvard.edu https://doi.org/

10.21542/gcsp.2020.39

Received: 22 November 2020 Accepted: 20 December 2020 (C) 2020 The Author(s), licensee Magdi Yacoub Institute. This is an open access article distributed under the terms of the Creative Commons Attribution license CC BY-4.0, which permits unrestricted use, distribution and reproduction in any medium, provided the original work is properly cited.
Parth N. Patel ${ }^{1}$, Robert P. Giugliano ${ }^{1,2 \star}$

\section{ABSTRACT}

Atherosclerotic cardiovascular disease (ASCVD) is highly prevalent and a major contributor to morbidity and mortality worldwide. Elevated blood cholesterol is a key driver of risk for atherosclerotic events, and patients with established ASCVD comprise a specific high-risk population in which low-density lipoprotein cholesterol (LDL-C) lowering therapy is strongly endorsed by multiple guidelines. An increasing number of medications across several pharmacologic classes are available today in clinical practice. Therefore, guidance on the appropriate use of these interventions is necessary for cost-effective solutions to managing residual atherothrombotic risk. In this review we summarize the key evidence supporting LDL-C lowering as described in the most recent 2018 multi-society Blood Cholesterol Guidelines, and provide a framework for optimizing LDL-C lowering therapy in secondary prevention populations. 


\section{INTRODUCTION}

Cardiovascular disease remains the leading cause of morbidity and mortality in the world and is estimated to account for one third of deaths globally each year ${ }^{1-3}$. Atherosclerotic cardiovascular disease (ASCVD), the dominant form of cardiovascular disease worldwide, is a chronic disorder of lipid deposition, vascular inflammation, and plaque formation that progresses sub-clinically before manifesting as ischemic stroke, myocardial infarction (MI), and peripheral limb ischemia. ASCVD events can be dramatic, at times leading to sudden and significant detriments to quality of life ${ }^{4,5}$. Fortunately, scientific advances have enhanced our understanding of the pathogenesis of ASCVD and have identified multiple risk factors responsible for its initiation and development over time. Clinical management of these risk factors has successfully decreased the burden of ASCVD over the last few decades ${ }^{6}$.

Elevated blood cholesterol is one of the primary causal risk factors for the development of ASCVD. Studies have shown that even modestly elevated levels of blood cholesterol increase rates of major adverse cardiac events ${ }^{7-9}$, and multiple guidelines support the use of cholesterol-lowering interventions in populations at elevated cardiovascular risk. Patients with a history of prior stroke, ischemic heart disease, or peripheral arterial disease comprise a specific population in which lipidlowering therapy is guideline supported for secondary prevention in all patients ${ }^{10}$. In this review we summarize the key evidence supporting the most recent 2018 multi-society Blood Cholesterol Guidelines ${ }^{10}$, with specific attention to treatments targeting low-density lipoprotein cholesterol, to provide a framework for optimizing lipid lowering therapy in patients with known prior ASCVD.

\section{Basics of the lipid panel}

A standard lipid panel provides total blood cholesterol levels as well as values for lipid subfractions. In general, the three main lipid subfractions of interest include low-density lipoprotein cholesterol (LDL-C), high-density lipoprotein cholesterol (HDLC), and triglycerides (TG). On a standard lipid panel, total cholesterol, HDL-C, and TG are all directly measured. By contrast, $L D L-C$ is usually calculated by the Friedwald formula ( $\mathrm{LDL}-\mathrm{C}=$ Total Cholesterol $-\mathrm{TG} / 5-\mathrm{HDL}-\mathrm{C})$, unless the triglycerides are markedly elevated ( $>4.5 \mathrm{mmol} / \mathrm{L}, 400 \mathrm{mg} / \mathrm{dL}$ ) or LDL-C is very low $(<1 \mathrm{mmol} / \mathrm{L}, 40 \mathrm{mg} / \mathrm{dL}$ ), in which case alternative formulas or methods are used ${ }^{11-13}$. Fasting and non-fasting lipid samples are equally adequate for most clinical applications ${ }^{14}$. However, in patients who demonstrate hypertriglyceridemia, a fasting lipid panel is recommended to confirm the diagnosis ${ }^{15,16}$.

Abnormalities of the lipid panel such as high LDL-C, low HDL-C, and high TG are all associated with greater ASCVD risk ${ }^{17-19}$. Several large cardiovascular outcomes trials have assessed the clinical benefit of modifying each type of lipid subfraction but have been met with varying results. Randomized controlled trials have repeatedly demonstrated that reduction of LDL-C decreases the incidence of cardiovascular events in both primary and secondary prevention cohorts ${ }^{20}$. On the other hand, attempts at increasing HDL-C with pharmacologic therapy have failed to show consistent benefit towards a composite cardiovascular endpoint ${ }^{21-27}$. Similarly, evidence supporting the use of TG-modifying therapies such as fibrates, niacin, or omega-3 fatty acids for ASCVD risk reduction has been mixed, especially in patients on baseline LDL-lowering therapy. High dose icosapent ethyl, a highly purified form of the omega-3 fatty acid eicosapentaenoic acid, has been shown to reduce major adverse cardiac events in statintreated patients with elevated TG and residual risk for ASCVD ${ }^{28}$. However, the benefits 
were independent of baseline or on-treatment TG levels suggesting the role of additional pleotropic effects of the drug ${ }^{29}$.

\section{Potency of LDL-C modifying therapies}

Given the robust data favoring $L D L-C$ reduction, guideline-based management of lipids in patients with established ASCVD is centered around LDL-C lowering therapies. The five standard LDL-C modifying medication classes available in clinical practice today include statins, ezetimibe, bile acid sequestrants, proprotein convertase subtilisin kexin type 9 (PCSK9) inhibitors, and bempedoic acid. These medication classes differ in their mechanism of action and their ability to achieve optimal LDL-C levels (Table 1).

Statins are competitive, reversible inhibitors of 3-hydroxy-3-methylglutaryl coenzyme A (HMG-CoA) reductase and continue to be the primary treatment modality for guidelinebased management of lipids in patients with ASCVD ${ }^{30}$. Through inhibiting HMG-CoA reductase, statins halt the rate limiting step of hepatic endogenous cholesterol production. A decrease in liver cholesterol leads to upregulation of the LDL-receptors on hepatocytes, facilitating endocytosis of LDL-C and its precursors from the systemic circulation, thereby reducing LDL-C levels in the bloodstream ${ }^{31}$.

Statin regimens can be subdivided into low-intensity, moderate-intensity, and highintensity subgroups based on the percent reduction of LDL-C achieved at that specific

Table 1 Percent of LDL-C-lowering provided by various lipid modifying therapies. Bempedoic acid is more likely to be used in statinintolerant patients and provides greater LDL-C percent reduction in this setting. A combination pill of bempedoic acid and ezetimibe is currently available that further lowers LDL-C by $35 \%$ in patients on stable background statin therapy.

\begin{tabular}{|c|c|c|}
\hline Treatment Category & Examples & LDL-C \% Reduction \\
\hline \multirow[t]{2}{*}{ High intensity statin } & Atorvastatin 40-80 mg daily & $\geq 50 \%$ \\
\hline & Rosuvastatin $20-40 \mathrm{mg}$ daily & \\
\hline \multirow[t]{7}{*}{ Moderate intensity statin } & Atorvastatin 10-20 mg daily & $30-49 \%$ \\
\hline & Fluvastatin 80 mg daily & \\
\hline & Lovastatin 40 mg daily & \\
\hline & Pitavastatin 2-4 mg daily & \\
\hline & Pravastatin 40-80 mg daily & \\
\hline & Rosuvastatin 5-10 mg daily & \\
\hline & Simvastatin 20-40 mg daily & \\
\hline \multirow[t]{5}{*}{ Low intensity statin } & Fluvastatin $20-40$ mg daily & $<30 \%$ \\
\hline & Lovastatin 20 mg daily & \\
\hline & Pitavastatin 1 mg daily & \\
\hline & Pravastatin 10-20 mg daily & \\
\hline & Simvastatin $10 \mathrm{mg}$ daily & \\
\hline Ezetimibe & Ezetimibe $10 \mathrm{mg}$ daily & $20-25 \%$ when added to baseline statin therapy \\
\hline \multirow[t]{2}{*}{ Bile Acid Sequestrants } & Colesevelam 3.75 g daily & $15 \%$ when added to baseline statin therapy \\
\hline & Cholestyramine 4-24 g daily & $15-25 \%$ when not receiving statin therapy \\
\hline \multirow[t]{2}{*}{ PCSK9 Inhibitor } & Evolocumab $140 \mathrm{mg}$ q2 weeks & $50-70 \%$ when added to baseline statin therapy \\
\hline & Alirocumab 75 mg q2 weeks & \\
\hline \multirow[t]{2}{*}{ Bempedoic Acid } & Bempedoic acid $180 \mathrm{mg}$ daily & $16-18 \%$ when added to baseline statin therapy \\
\hline & & $20-22 \%$ when not receiving statin therapy \\
\hline $\begin{array}{l}\text { Bempedoic Acid + } \\
\text { Ezetimibe Combination }\end{array}$ & Bempedoic acid $180 \mathrm{mg}+$ Ezetimibe $10 \mathrm{mg}$ daily & $35 \%$ when added to baseline statin therapy \\
\hline Inclisiran & Inclisiran $300 \mathrm{mg}$ on day 1 , day 9o, then q6 months & $50 \%$ when added to baseline statin therapy \\
\hline
\end{tabular}


dose and formulation. Most of the LDL-C reducing effect is seen with the initial starting dose. Each additional doubling of the dose of any statin only provides an additional $6 \%$ reduction in LDL-C ${ }^{32}$. For example, though $10 \mathrm{mg}$ of atorvastatin reduces LDL-C by $37 \%$, sequential doubling of this dose to $20 \mathrm{mg}, 40 \mathrm{mg}$, and $80 \mathrm{mg}$ provides $43 \%, 49 \%$, and $55 \% \mathrm{LDL}-\mathrm{C}$ reduction, respectively. There are at least two likely explanations for the decreased efficacy with higher doses. First, as endogenous cholesterol biosynthesis is inhibited, there is a compensatory increase in exogenous cholesterol absorption through the gastrointestinal tract ${ }^{33}$. Second, statin treatment induces production of PCSK9, a key protein that binds hepatic LDL receptors, triggers receptor degradation, and prevents clearance of LDL-C particles from the circulation ${ }^{34}$.

Ezetimibe, an oral selective cholesterol absorption inhibitor, is the most commonly used non-statin agent. Combined treatment with statin and ezetimibe synergistically targets both the endogenous and exogenous pathways of cholesterol metabolism. When added to baseline statin therapy, ezetimibe provides an additional $20-25 \%$ reduction in $\mathrm{LDL}-\mathrm{C} 33,35$. Whereas $80 \mathrm{mg}$ atorvastatin provides $55 \% \mathrm{LDL}-\mathrm{C}$ reduction, a similar reduction can be achieved by $10 \mathrm{mg}$ atorvastatin plus $10 \mathrm{mg}$ ezetimibe.

Bile acid sequestrants, oral polymers that impede the reabsorption of bile salts in the small intestine, represent another non-statin alternative that have proven LDL-C lowering efficacy. Depending on the dose prescribed, bile acid sequestrants reduce LDL-C levels by $15-2 \%^{36-38}$, and are felt to have a favorable safety profile due to a lack of meaningful absorption from the gut into the bloodstream. However, gastrointestinal complaints, high pill burden, drug-drug interactions, and lack of outcomes data in the statin era have limited the use of this class of medications in modern clinical practice.

PCSK9 inhibitors prevent the PCSK9 protein from binding to the LDL receptor, thereby interrupting attempts at LDL receptor degradation and allowing for increased clearance of LDL-C from the bloodstream. The currently available PCSK9 inhibitors are injectable monoclonal antibodies that markedly reduce LDL-C and are increasingly used in high-risk patients. In statin-treated patients, PCSK9 inhibitors have been shown to reduce LDL-C levels by an additional 50-70\%39,40.

Bempedoic acid was granted approval by the US Food and Drug Administration (FDA) in February 2020 as an adjunct to maximally tolerated statin therapy in adults with familial hypercholesterolemia or established ASCVD who require additional lowering of LDL-C ${ }^{41}$. Bempedoic acid is an oral prodrug that undergoes liver-specific activation to inhibit adenosine triphosphate-citrate lyase, an important enzyme in the cholesterol biosynthesis pathway directly upstream of HMG-CoA reductase. In multiple phase 3 trials, treatment with bempedoic acid has been shown to lower LDL-C levels by $16-18 \%$ in patients on maximally tolerated statin therapy ${ }^{42-45}$. A combination of bempedoic acid and ezetimibe reduces LDL-C by approximately $35 \%{ }^{46}$, and is a promising alternative in patients who are intolerant of statins.

\section{Evidence base for LDL-modifying therapies}

Clear evidence supporting reduction of LDL-C with statins to lower the incidence of major cardiovascular events comes from a 2010 meta-analysis performed by the Cholesterol Treatment Trialists' (CTT) Collaboration. Given the understanding that small sample sizes, inadequate event rates, and short follow up are all likely to lead to underpowered and negative individual studies, the CTT Collaboration was founded to meta-analyze all randomized controlled trials of statin therapy that studied at least 1,000 patients over 2 years of treatment with the aim of quantifying the effect of LDL-C lowering on major cardiovascular events. 


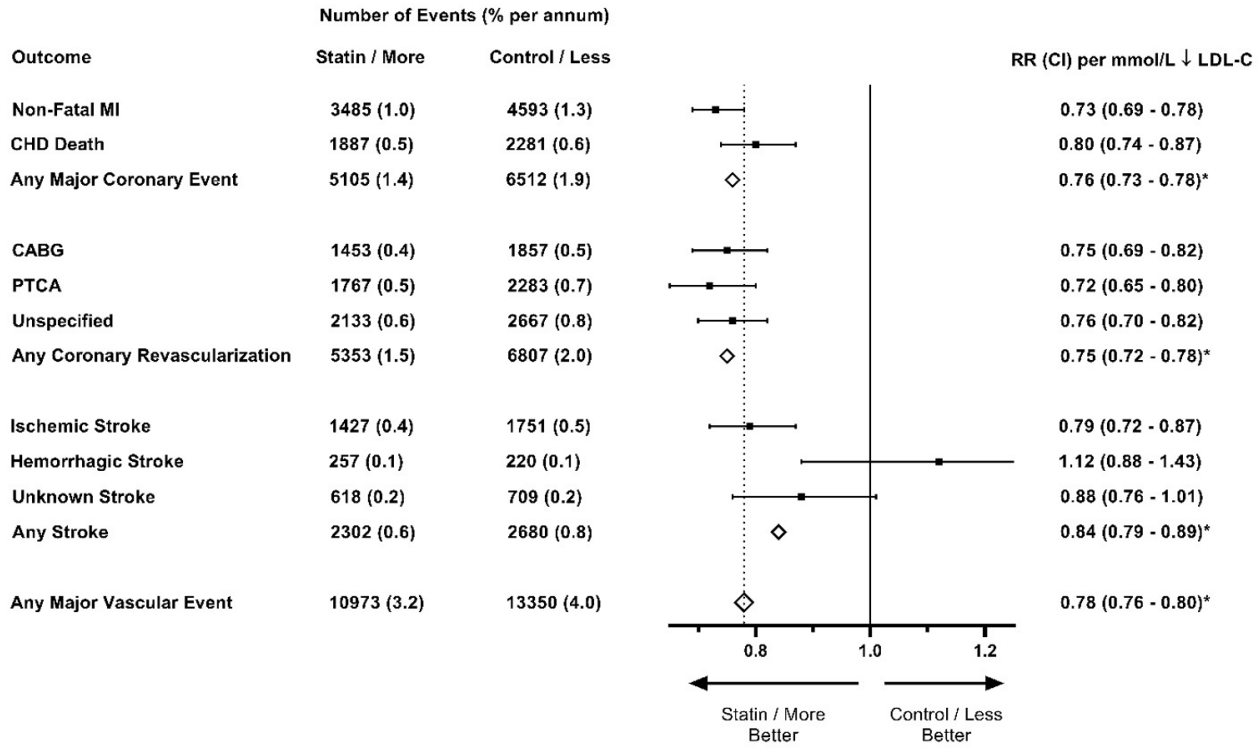

Figure 1. Summary of major vascular event endpoints in CTT Collaboration per one $\mathrm{mmol} / \mathrm{L}$ reduction in LDL-C. Adapted from the CTT Collaboration website (https://www.cttcollaboration.org/). Each one $\mathrm{mmol} / \mathrm{L}$ reduction in $\mathrm{LDL}-\mathrm{C}$ was associated with significant relative risk reductions in major coronary events (24\%), coronary revascularizations (25\%), and stroke (16\%). Altogether, there was a $22 \%$ relative risk reduction across all major vascular events per one $\mathrm{mmol} / \mathrm{L}$ reduction in LDL-C. All confidence intervals are $95 \%$ confidence intervals unless indicated by an asterisk, in which a $99 \%$ confidence interval is shown. $\mathrm{MI}=$ Myocardial Infarction. $\mathrm{CHD}=$ Coronary Heart Disease. $\mathrm{CABG}=$ Coronary Artery Bypass Grafting. PTCA $=$ Percutaneous Transluminal Coronary Angioplasty. RR $=$ Relative Risk. $\mathrm{Cl}=$ Confidence Interval. LDL-C $=$ Low-Density Lipoprotein Cholesterol.

In an analysis of 26 randomized trials involving nearly 170,000 individuals, major vascular event rates were directly related to the absolute reduction in LDL-C that was achieved ${ }^{20}$. Specifically, each one $\mathrm{mmol} / \mathrm{L}(\sim 38.7 \mathrm{mg} / \mathrm{dL})$ reduction in $\mathrm{LDL}-\mathrm{C}$ with statin therapy produced a $22 \%$ proportional reduction in the rate of major vascular events over a median follow up of five years (Figure 1). This finding was remarkably consistent across baseline LDL-C and various subgroups, including those with and without prior ASCVD. Moreover, across all trials, the absolute reduction in LDL-C levels while on treatment accounted for $98 \%$ of the between-study variability in relative risk reduction 47 .

This same relationship between achieved reduction in LDL-C and relative risk reduction for ASCVD events has been shown to be equally true for non-statin therapies. In a meta-analysis of eight trials studying diet, ezetimibe, bile acid sequestrants, and ileal bypass, each one $\mathrm{mmol} / \mathrm{L}$ reduction in LDL-C was associated with a $25 \%$ relative risk reduction in cardiovascular event rates ${ }^{47}$. Though similar analyses of PCSK9 inhibitor trials are limited by short duration of follow up, PCSK9 inhibitor treatment provides consistent effects on the risk of cardiovascular events per mmol/L reduction in LDL-C when directly comparing the first years of treatment ${ }^{48,49}$.

Implicit in this finding is the fact that if all individuals receive an equivalent relative risk reduction per $\mathrm{mmol} / \mathrm{L}$ reduction in $\mathrm{LDL}-\mathrm{C}$, then those with higher absolute risk are most likely to receive the greater absolute benefit. For example, a low risk, young individual without cardiovascular risk factors with a 10-year ASCVD risk of $1 \%$ would reduce their risk to approximately $0.78 \%$ risk if they achieved one $\mathrm{mmol} / \mathrm{L} \mathrm{LDL-C} \mathrm{lowering} \mathrm{over} \mathrm{the}$ time period studied. A higher risk, older individual with cardiovascular comorbidities at 
$30 \%$ baseline 10-year risk experiencing an equivalent LDL-C reduction would have $23.4 \%$ risk over the same period and receive a greater absolute benefit (0.22\% vs. 6.6\% per decade).

Additional data suggest that the relative risk reduction provided by LDL-C-lowering is even greater when initiated earlier in life. Mendelian randomization studies of individuals who inherit an LDL-C lowering allele show that long-term exposure to lower LDL-C through a genetic mutation is associated with a three-fold greater reduction in the risk of ASCVD per one $\mathrm{mmol} / \mathrm{L}$ decrease in $\mathrm{LDL}-\mathrm{C}$ when compared to the same degree of LDL-C-lowering in adulthood ${ }^{50}$. These data suggest that LDL-C has not only a causal but possibly a cumulative effect on the risk of ASCVD. Taken together, reducing LDL-C levels leads to a dose-dependent decrease in the risk of major ASCVD events that is directly proportional to the absolute magnitude of reduction achieved in LDL-C, with a possible larger effect seen over larger periods of time ${ }^{51}$.

\section{Guideline-based management of lipids in secondary prevention of atherosclerotic cardiovascular disease}

The 2018 multi-society Blood Cholesterol Guidelines define clinical ASCVD as an all-encompassing term for several diseases of atherosclerotic origin ${ }^{10}$. Patients with acute coronary syndromes, prior MI, stable angina, stroke, transient ischemic attack, peripheral artery disease, or history of any arterial revascularization due to atherosclerotic disease are at very high risk for future ischemic events. All patients with clinical ASCVD are recommended to be on lipid lowering therapy.

\section{All patients with ASCVD should be considered for maximally tolerated statin therapy}

In patients with clinical ASCVD $\leq 75$ years of age, high-intensity statin therapy is recommended with the goal of achieving a $50 \%$ or greater reduction in LDL-C levels. If high-intensity statin therapy is contraindicated or limited by side effects, a moderate-intensity statin is supported.

In the CTT Collaboration meta-analysis, there were 21 trials that compared statin versus control, and another five trials that compared more versus less intensive statin regimens ${ }^{20}$. Among the 21 trials that assessed allocation to routine statin therapy versus no statin therapy, the average reduction in LDL-C was approximately one $\mathrm{mmol} / \mathrm{L}$, which resulted in a $22 \%$ relative risk reduction in major cardiovascular events. In five trials comparing more (i.e., high-intensity) versus less intensive (i.e., moderate-intensity) statin therapy, the mean additional reduction in LDL-C was $0.51 \mathrm{mmol} / \mathrm{L}(19.7 \mathrm{mg} / \mathrm{dL})$, which translated into a further $15 \%$ relative risk reduction in major vascular events.

Combining the evidence in the intention-to-treat analyses of these two groups of trials revealed that allocation to a high-intensity statin regimen reduces LDL-C by approximately $1.5 \mathrm{mmol} / \mathrm{L}(58.4 \mathrm{mg} / \mathrm{dL})$ when compared to no statin therapy ${ }^{52}$. Therefore, it is estimated that treatment with a high-intensity statin provides a $34 \%$ relative risk reduction for major vascular events over the time period studied $(0.78 \times$ $0.85=0.66$ ). In terms of absolute risk, an individual with prior ASCVD at baseline $20 \%$ five-year risk of a major vascular event would have a reduction in the predicted 5-year event rate to $13 \%(20 \times 0.66)$ if treated with a high-intensity statin, or to $16 \%(20 \times 0.78)$ if treated with a moderate-intensity statin (Figure 2 ). Based on these data, guidelines support the initiation of the highest intensity statin tolerated.

In patients with age greater than 75 years, the 2018 multi-society Blood Cholesterol Guidelines recommend initiation of a moderate or high intensity statin after weighing these benefits against adverse effects ${ }^{10}$. In contrast, the 2019 European Society of 


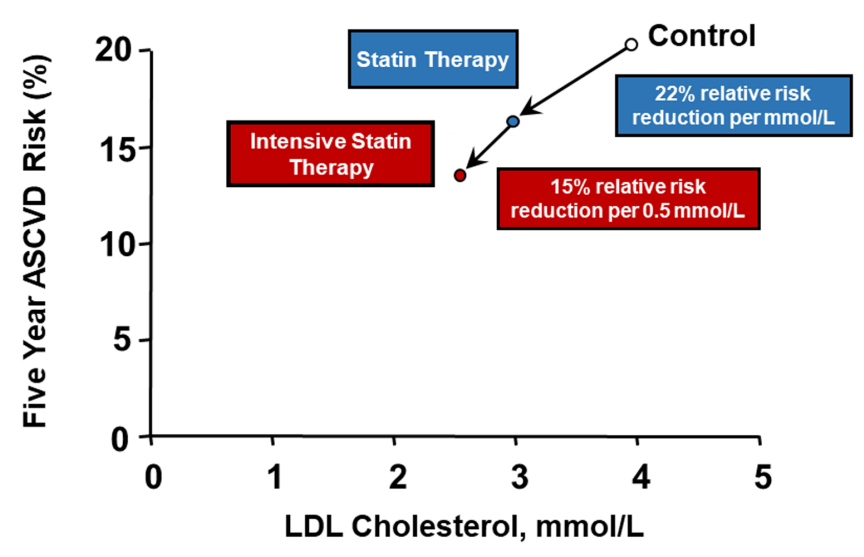

Figure 2. High intensity statin therapy provides additional relative risk reduction to moderate intensity statin therapy. Adapted from the CT Collaboration website (https://www.cttcollaboration. org/). On average, allocation to moderate-intensity statin therapy reduces LDL-C by approximately one $\mathrm{mmol} / \mathrm{L}$ and leads to a $22 \%$ relative risk reduction in major vascular events. Treatment with an intensive (i.e., high-intensity) statin regimen leads to an additional $0.5 \mathrm{mmol} / \mathrm{L}$ reduction in $\mathrm{LDL}-\mathrm{C}$ and further lowers the risk of major vascular events by an additional $15 \%$. It follows that a patient with $20 \%$ baseline five year ASCVD risk would reduce risk to 16\% (20 ×0.78) with moderate-intensity statin therapy, or $13 \%(20 \times 0.78 \times 0.85)$ with high-intensity statin therapy over the time period studied. In patients with prior ASCVD, guidelines support maximal risk reduction with the most intensive statin treatment tolerated.

Cardiology (ESC)/European Atherosclerosis Society (EAS) Guidelines recommend treatment with the highest intensity statin tolerated across all age ranges ${ }^{53}$. The primary reason behind this discrepancy had been the observation in the CTT Collaboration data that the relative reduction in major vascular events per one $\mathrm{mmol} / \mathrm{L} L \mathrm{LD}-\mathrm{C}$ reduction was possibly lower among patients aged $>75$ years 54 . However, in a meta-analysis of

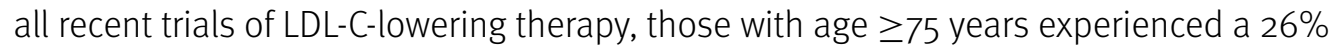
relative risk reduction per one $\mathrm{mmol} / \mathrm{L}$ lowering of $\mathrm{LDL}-\mathrm{C}$, with no significant difference from those younger than 75 years ${ }^{55}$. Given this clearer data that LDL-C lowering is as effective in reducing major cardiovascular events in patients aged 75 years or older, and the knowledge that the absolute risk of ASCVD rises with increasing age, it is expected that future guidelines will strengthen recommendations for lipid lowering in this older population.

\section{Ezetimibe should be considered in patients with ASCVD and LDL-C Level of $1.8 \mathrm{mmol} / \mathrm{L}$ (70 $\mathrm{mg} / \mathrm{dL})$ or higher}

In patients with prior ASCVD who continue to have elevated LDL-C levels on statin monotherapy, adjunctive therapy with ezetimibe is recommended. The addition of ezetimibe to baseline statin therapy provides additional reduction in LDL-C and is a Class II recommendation in the Blood Cholesterol Guidelines. Ezetimibe use in this population is supported by the results of The Improved Reduction of Outcomes: Vytorin Efficacy International Trial (IMPROVE-IT). In IMPROVE-IT, 18,144 patients with ACS in the prior 10 days who had LDL-C levels of $1.3-2.6 \mathrm{mmol} / \mathrm{L}(50-100 \mathrm{mg} / \mathrm{dL})$ on lipid lowering therapy or $1.3-3.2 \mathrm{mmol} / \mathrm{L}(50-125 \mathrm{mg} / \mathrm{dL})$ without lipid lowering therapy were randomized to receive either combination simvastatin 40 mg-ezetimibe $10 \mathrm{mg}$ versus simvastatin 40 $\mathrm{mg}$ alone ${ }^{56}$. The primary endpoint was a composite of cardiovascular death, nonfatal MI, nonfatal stroke, unstable angina requiring hospitalization, and coronary revascularization greater than 30 days after randomization. 
At time of hospitalization for the index event, the mean LDL-C was $2.4 \mathrm{mmol} / \mathrm{L}$ (93.8 $\mathrm{mg} / \mathrm{dL}$ ) in both groups. Patients in the combined simvastatin-ezetimibe arm had a median time-weight average LDL-C of $1.4 \mathrm{mmol} / \mathrm{L}(53.7 \mathrm{mg} / \mathrm{dL})$ whereas those in the simvastatin alone arm had a median time-weighted average LDL-C of $1.8 \mathrm{mmol} / \mathrm{L}$ (69.5 $\mathrm{mg} / \mathrm{dL}$ ). At seven years, the primary endpoint occurred less frequently in the combined treatment arm versus the statin monotherapy arm (32.7\% vs. 34.7\%, HR 0.936, 95\% $\mathrm{Cl}$ [0.89-0.99], $p=0.016$, number needed to treat $=50$ ). The relative risk reduction in major cardiovascular events provided by ezetimibe was consistent with the prior $\mathrm{CT}$ Collaboration finding of $22 \%$ reduction in major vascular events per one $\mathrm{mmol} / \mathrm{L}$ reduction in $\mathrm{LDL}-\mathrm{C}$, as the $\mathrm{HR}$ for clinical benefit per one $\mathrm{mmol} / \mathrm{L} \mathrm{LDL}-\mathrm{C}$ reduction in the study was 0.80 . Of note, whereas the average baseline LDL-C level in the CT Collaboration meta-analysis was $3.4 \mathrm{mmol} / \mathrm{L}$ (131 mg/dL), IMPROVE-IT demonstrated that the magnitude of clinical benefit from further LDL-C lowering was similar even at lower starting ranges of LDL-C. Furthermore, cardiovascular event rates were lowest in those who achieved the lowest LDL-C levels, with no observed excess in safety events even among those who achieved an LDL-C $<0.8 \mathrm{mmol} / \mathrm{L}(30 \mathrm{mg} / \mathrm{dL})$ and were followed for 6 years 57 .

In IMPROVE-IT, there was a broad spectrum of risk in the trial with regard to the incidence of recurrent ischemic events. Patients with a greater burden of atherothrombotic risk factors not only had higher event rates, but also experienced greater benefit from ezetimibe, whereas those with fewer risk factors had lesser reductions in major cardiovascular outcomes ${ }^{58}$. High risk subgroups independently shown to have significantly larger benefits with ezetimibe than the rest of the study population included age $\geq 75$ years ${ }^{59}$, prior stroke ${ }^{60}$, prior coronary artery bypass grafting $^{61}$, diabetes ${ }^{62}$, and peripheral arterial disease ${ }^{63}$. As such, the most recent Blood Cholesterol Guidelines make a Class Ila recommendation for initiating ezetimibe in those with prior ASCVD, LDL-C greater than $1.8 \mathrm{mmol} / \mathrm{L}(70 \mathrm{mg} / \mathrm{dL})$ on maximally tolerated statin therapy, and very high atherothrombotic risk due to risk-enhancing features. As the benefit of ezetimibe was less apparent in low risk trial participants, ezetimibe therapy in all individuals with prior ASCVD has received a weaker Class Ilb recommendation.

\section{PCSK9 inhibitors should be considered in high-risk patients with ASCVD on maximal LDL-lowering therapy and LDL level of $1.8 \mathrm{mmol} / \mathrm{L}(70 \mathrm{mg} / \mathrm{dL})$ or higher}

In patients with prior ASCVD who are unable to achieve LDL-C levels of $1.8 \mathrm{mmol} / \mathrm{L}$ (70 mg/dL) with statins and ezetimibe, treatment with PCSK9 inhibitors is guideline supported for the highest risk patients. The data for intensive lipid lowering with PCSK9 inhibitors comes from two large cardiovascular outcomes trials. The two agents studied in these trials, evolocumab and alirocumab, are fully human monoclonal antibodies administered subcutaneously and have been approved for clinical use ${ }^{49}$.

The Further Cardiovascular Outcomes Research with PCSK9 Inhibition in Subjects with Elevated Risk (FOURIER) trial was the first successfully completed cardiovascular outcomes trial of PCSK9 inhibition. In FOURIER, 27,564 high-risk patients with established cardiovascular disease, defined as prior MI, prior non-hemorrhagic stroke, or symptomatic PAD, were recruited into a lipid stabilization phase which included intensive statin therapy with or without ezetimibe ${ }^{64}$. Those on maximal medical therapy who continued to have LDL-C $\geq 1.8 \mathrm{mmol} / \mathrm{L}$ (70 mg/dL) or non-HDL-C $\geq 2.6 \mathrm{mmol} / \mathrm{L}(100$ $\mathrm{mg} / \mathrm{dL}$ ) were then randomly assigned to receive evolocumab or matching placebo injections. The primary endpoint was a composite of major cardiovascular events. The median duration of follow up was 2.2 years. 
The median baseline plasma LDL-C level for the study was $2.4 \mathrm{mmol} / \mathrm{L}(92 \mathrm{mg} / \mathrm{dL})$. Evolocumab lowered LDL-C levels by $59 \%$ at 48 weeks compared to placebo, with a median on-treatment level of $0.8 \mathrm{mmol} / \mathrm{L}$ [IQR 0.5 to $1.2,30 \mathrm{mg} / \mathrm{dL}$ (IQR: 19 to 46)], a reduction that was maintained over the duration of the study. The evolocumab treatment group had a significantly lower incidence of the primary composite endpoint of cardiovascular death, nonfatal MI, nonfatal stroke, hospitalization for unstable angina, or coronary revascularization when compared to placebo ( $9.8 \%$ vs $11.3 \%$; HR 0.85, $95 \%$ $\mathrm{Cl}$ [0.79-0.92], $p<0.001)$. The benefit of evolocumab was consistent across all key subgroups, including the patients in the lowest quartile of baseline LDL-C levels $(<1.9$ $\mathrm{mmol} / \mathrm{L}, 74 \mathrm{mg} / \mathrm{dL}$ ) and those on maximal dose statin ${ }^{65}$. Moreover, on-treatment LDL$C$ at four weeks was highly predictive of risk for the primary and secondary composite endpoints, with incremental risk reduction conferred to subgroups that achieved lower targets ( $p$-trend $<0.0001$, Figure 3$)^{66}$.

ODYSSEY OUTCOMES was a second randomized controlled trial of PCSK9 inhibition that studied 18,924 patients with recent acute coronary syndromes in the prior 1 to 12 months ${ }^{67}$. Patients first underwent a run-in period involving stabilization on the highest tolerated dose of atorvastatin or rosuvastatin. Those with $L D L-C \geq 1.8 \mathrm{mmol} / \mathrm{L}(7 \mathrm{O} \mathrm{mg} / \mathrm{dL})$, non-HDL-C $\geq 2.6 \mathrm{mmol} / \mathrm{L}(100 \mathrm{mg} / \mathrm{dL})$, or apolipoprotein $B \geq 2.1 \mathrm{mmol} / \mathrm{L}(80 \mathrm{mg} / \mathrm{dL})$ while on maximally tolerated statin treatment qualified for randomization to either alirocumab or placebo subcutaneous injections every two weeks. Subjects assigned to the alirocumab treatment arm underwent a blinded dose-adjustment protocol to target an $\mathrm{LDL}-\mathrm{C}$ range of $0.6-1.3 \mathrm{mmol} / \mathrm{L}(25-50 \mathrm{mg} / \mathrm{dL})$ during this study. Though on-treatment LDL-C levels $\leq 0.6 \mathrm{mmol} / \mathrm{L}(25 \mathrm{mg} / \mathrm{dL}$ ) were permitted, patients who achieved sustained LDL-C levels below $0.4 \mathrm{mmol} / \mathrm{L}(15 \mathrm{mg} / \mathrm{dL}$ ) while on the lowest dose of alirocumab were switched to placebo. Patients were followed for a median of 2.8 years for the primary composite endpoint of coronary-related death, nonfatal MI, nonfatal ischemic stroke, or unstable angina requiring hospitalization.

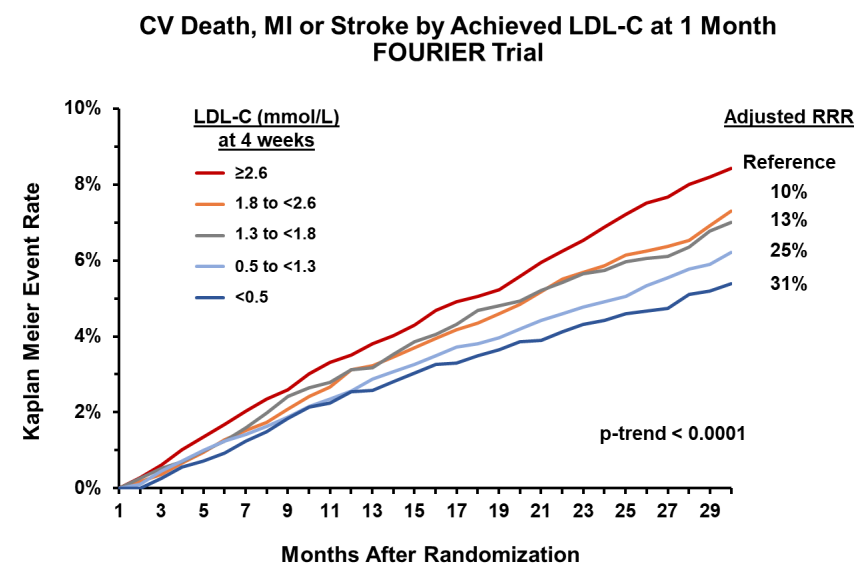

Figure 3. Kaplan-Meier event rate of cardiovascular death, myocardial infarction or stroke in FOURIER trial by achieved LDL-C at 4 weeks. In the FOURIER trial[ ${ }^{[64]}$, the achieved LDL-C at four weeks following randomization was significantly associated with incidence of the key secondary endpoint, a composite of cardiovascular death, myocardial infarction, or stroke. Groups that achieved lower LDL-C targets obtained greater relative risk reduction ( $p$-trend $<0.0001$ ). The group of subjects that achieved LDL-C $<0.5 \mathrm{mmol} / \mathrm{L}(20 \mathrm{mg} / \mathrm{dL}$ ) had the lowest incidence of the combined endpoint, and had a 31\% relative risk reduction compared to the reference group ( $\mathrm{LDL} \geq 2.6 \mathrm{mmol} / \mathrm{L}, 100 \mathrm{mg} / \mathrm{dL}$ ) over the trial study period. $\mathrm{CV}=$ Cardiovascular. $\mathrm{MI}=$ Myocardial Infarction. $\mathrm{LDL}-\mathrm{C}=$ Low-Density Lipoprotein Cholesterol. RRR = Relative Risk Reduction. 
The mean LDL-C across both treatment groups at baseline was $2.4 \mathrm{mmol} / \mathrm{L}$ (92 $\mathrm{mg} / \mathrm{dL}$ ). When compared to placebo, alirocumab treatment reduced LDL-C levels by $63 \%$, $61 \%$, and $55 \%$ at 4,12 , and 48 months, respectively, among those who remained on treatment. In an intention-to-treat analysis, the composite primary endpoint occurred less frequently in the alirocumab treatment arm compared to placebo ( $9.5 \%$ vs $11.1 \%$, $\mathrm{HR} 0.85,95 \% \mathrm{Cl}$ [0.78-0.93], $p<0.001$ ). Subgroup analyses demonstrated that those who had a baseline $L D L-C \geq 2.6 \mathrm{mmol} / \mathrm{L}(100 \mathrm{mg} / \mathrm{dL})$ may have had a more prominent absolute risk reduction than those with lower baseline LDL-C levels. However, due to the protocol mandated dose-adjustment algorithm, in which patients with lower baseline LDL-C levels were more likely to receive the lower dose of alirocumab and more likely to have alirocumab stopped completely due to very low achieved LDL-C, the observation that subgroups with lower LDL-C levels at baseline received a diminishing degree of LDL$C$ reduction is likely to be confounded.

From the results of these two cardiovascular outcomes trials, the 2018 Blood Cholesterol Guidelines recommend consideration of PCSK9 inhibitors in high-risk patients with prior ASCVD events on maximally tolerated LDL-C lowering therapy and LDL $\geq 1.8$ $\mathrm{mmol} / \mathrm{L}(70 \mathrm{mg} / \mathrm{dL})$. The decision to initiate treatment should weigh the expected benefit towards major adverse cardiovascular events against the expenses incurred from being on treatment (approximately $\$ 6,000 /$ year in the USA), though expected future reductions in the price of these drugs will allow this therapy to be more cost-effective with time ${ }^{68}$. Additionally, though five year follow up from an open-label extension of phase 2 studies with evolocumab raised no new safety concerns, longer-term safety data from randomized trials are still needed ${ }^{69}$. Fortunately, two ongoing trials of PCSK9 inhibition with planed four to five-year follow up are underway (discussed below).

\section{Bempedoic acid may emerge as an alternative in statin-intolerant patients}

Given the incorporation of non-statin LDL-C-lowering therapies into the professional guidelines, recent attention has been directed to bempedoic acid, a first-in-class orally administered ATP citrate lyase inhibitor which offers promise for additional LDL-C reduction in patients on maximally tolerated lipid-lowering therapy. Since the publication of the 2018 Blood Cholesterol Guidelines, several phase 3 lipid-lowering trials have shown that bempedoic acid significantly lowers LDL-C levels when compared to placebo in at-risk populations for which additional LDL-C lowering is recommended. While cardiovascular outcomes data for this drug class are not yet available, two large trials of bempedoic acid designed to study intermediate endpoints, CLEAR Harmony ${ }^{42}$ and CLEAR Wisdom ${ }^{43}$, had promising results reported in 2019.

CLEAR Harmony was a multicenter, randomized, placebo-controlled trial of 2,230 patients with either prior ASCVD, heterozygous familial hypercholesterolemia, or both and an LDL-C concentration of at least $1.8 \mathrm{mmol} / \mathrm{L}(70 \mathrm{mg} / \mathrm{dL})$ while on maximallytolerated lipid lowering therapy ${ }^{42}$. At enrollment, nearly all patients were on a statin and $7.7 \%$ were receiving treatment with ezetimibe. The mean LDL-C at baseline across the entire trial cohort was $2.7 \mathrm{mmol} / \mathrm{L}(103.2 \mathrm{mg} / \mathrm{dL}$ ). Patients were randomized in a 2:1 fashion to treatment with $180 \mathrm{mg}$ bempedoic acid daily or matching placebo for a total of 52 weeks. The primary endpoint was safety and the principal efficacy endpoint was the percentage change in LDL-C levels at 12 weeks. At week 12, treatment with bempedoic acid reduced LDL-C by $18.1 \%$ when compared to the placebo group ( $95 \% \mathrm{Cl}-20.0 \%$ to $-16.1 \%, p<0.001$ ). This effect did not attenuate over the 52 -week trial period.

CLEAR Wisdom was a similarly designed multicenter, randomized, placebo-controlled trial of 779 patients with prior ASCVD, heterozygous familial hypercholesterolemia, or 
both with an LDL-C concentration of at least $2.6 \mathrm{mmol} / \mathrm{L}(100 \mathrm{mg} / \mathrm{dL})$ while on maximallytolerated lipid lowering therapy at the first screening visit43. Unlike CLEAR Harmony, which was designed for a primary safety endpoint, CLEAR Wisdom was designed to assess the primary end point of percent change from baseline in LDL-C level at week 12. Most patients enrolled (89.6\%) were on background statin therapy, but $5 \%$ of participants were not taking any lipid-lowering therapy at baseline. The mean LDL-C concentration at randomization was $3.1 \mathrm{mmol} / \mathrm{L}(120.4 \mathrm{mg} / \mathrm{dL})$. Patients were randomized 2:1 to treatment with bempedoic acid or matching placebo. At week 12, treatment with bempedoic acid resulted in a $17.4 \%$ decrease in LDL-C concentration compared to placebo (95\% Cl $-21.0 \%$ to $-13.9 \%, p<0.001$ ). Additionally, bempedoic acid reduced levels of high-sensitivity C-reactive protein (hsCRP) throughout the study, consistent with other preclinical studies of the drug ${ }^{70,71}$.

Based on these data, bempedoic acid was granted FDA approval in February 2020 as an adjunctive treatment for patients with familial hypercholesterolemia or established ASCVD who require additional lowering of LDL-C. It is important to note that neither CLEAR Harmony nor CLEAR Wisdom was powered to study the effect of bempedoic acid on cardiovascular outcomes, so the expected clinical benefit of bempedoic acid is currently based on extensive prior data demonstrating a consistent linear relationship between achieved LDL-C levels and subsequent cardiovascular risk. While it is notable that adjudicated major adverse cardiovascular event rates were lower in the bempedoic acid treatment arms in both trials, these were trends that did not reach statistical significance. CLEAR Outcomes, a large phase 3 clinical outcomes trial with bempedoic acid, is ongoing with results anticipated in 2022-23 (discussed below) ${ }^{72}$.

\section{Lipoprotein apheresis can be considered for additional LDL-C lowering in patients who have inadequate response to available therapies}

In high-risk patients in whom the above therapeutic options are not tolerated, or ineffective in achieving target LDL-C levels, lipoprotein apheresis remains a highlyeffective rescue treatment for severe dyslipidemia ${ }^{73}$. In lipoprotein apheresis, the extracorporeal removal of circulating apoB-containing lipoproteins acutely reduces LDL-C levels, lowering LDL-C by $60-80 \%$ when compared to concentrations immediately prior to the procedure. However, given the transient nature of these LDL-C reductions, serial treatments every one or two weeks are necessary to achieve the degree of time-averaged lowering of LDL-C desired ${ }^{74,75}$.

Apheresis represents a highly-specialized, time-intensive, and expensive endeavor to achieve LDL-C targets in patients, with costs estimated at $\$ 50,000$ per year ${ }^{76}$. For these reasons, extracorporeal therapy is usually reserved for patients with homozygous familial hypercholesterolemia in whom lipid-lowering drugs are usually less effective, and LDL-C reduction can be life-saving77,78. Lipoprotein apheresis is also offered to patients with heterozygous or severe forms of hypercholesterolemia, in whom maximal lipid-lowering therapy only reduces $L D L-C$ to levels of $5.2-7.8 \mathrm{mmol} / \mathrm{L}(200-300 \mathrm{mg} / \mathrm{dL})^{79,80}$. The LDL-C level at which to initiate treatment varies by country and is usually influenced by whether ASCVD is present. As such, the need for lipoprotein apheresis is often determined by specialists on a case-by-case basis.

The reason behind such varied practice internationally is due to the lack of randomized longitudinal outcomes data for lipoprotein apheresis. Observational studies comparing lipoprotein apheresis to standard oral drug therapy have suggested that extracorporeal treatment reduces a composite cardiovascular endpoint by $72 \%$ over a six year follow up period ${ }^{81}$. Retrospective analyses comparing the burden of ASCVD before 
and after initiation of apheresis therapy have suggested a reduction in cardiovascular events between $30-80 \%$ in the time period on apheresis treatment ${ }^{82,83,84}$.

Though lipoprotein apheresis has had a larger role in prior decades, recently approved treatments such as PCSK9 inhibitors may decrease the necessity of apheresis in contemporary practice. Data supporting this claim comes from the ODYSSEY ESCAPE trial, a double-blind, randomized, placebo-controlled trial of PCSK9 inhibition in patients with heterozygous familial hypercholesterolemia who were undergoing weekly or biweekly lipoprotein apheresis ${ }^{85}$. In this trial, a total of 62 subjects were randomly assigned in a 2:1 fashion to alirocumab 150 mg every 2 weeks or matching placebo for an 18 week follow up period. In weeks 1 through 6, the apheresis rate was fixed while patients initiated the randomly assigned treatment. In weeks 7 through 18, apheresis treatments were adjusted based on the LDL-C response in a blinded manner, with complete discontinuation of apheresis if the LDL-C was reduced by $30 \%$ or more from baseline. The primary efficacy endpoint was the change in rate of apheresis treatments over a 12-week (weeks 7 through 18) follow up period.

At the end of week 6, the change in LDL-C from baseline was $-53.7 \%(95 \% \mathrm{Cl}-58.2$ to -49.2$)$ in the alirocumab group and $1.6 \%(95 \% \mathrm{Cl}-4.7$ to 7.9$)$ in the placebo group. From weeks 7 through 18 , patients assigned to alirocumab experienced a $75 \%$ reduction in the standardized rates of apheresis when compared to placebo-treated patients ( $p<$ 0.0001 ). More specifically, $90 \%$ of patients on alirocumab halved the rate of apheresis over the 12 -week follow up period, while $63.4 \%$ of patients on alirocumab avoided apheresis altogether. Based on the results of this study, PCSKg inhibitors may play an increasing role in patients with heterozygous familial hypercholesteremia undergoing routine apheresis therapy. Furthermore, with the recent approval of bempedoic acid, and many other investigational LDL-C lowering therapeutics in development, it is possible that the need for apheresis may be decreased further in the coming years.

\section{Safety of LDL-C lowering therapies}

Although the benefits of LDL-C lowering therapies are well documented, no drug class is without adverse effects and all decisions regarding initiation of treatment should weigh the risks and benefits for individual patients.

Statins cause a small absolute excess of muscle related adverse events (approximately 1 case per 10,000 individuals treated per year) ${ }^{52,86,87}$, with increased rates at higher doses of statins and when used concurrently with drugs that affect their metabolism ${ }^{88}$. In observational studies, statin treatment has been associated with a higher rate of symptomatic muscle pain or weakness in roughly $5 \%$ of patients ${ }^{89,90}$, but these rates have been shown to be equivalent in placebo-treated patients across numerous randomized controlled trials ${ }^{91,92}$. It is also estimated that standard statin regimens increase the risk of incident diabetes by approximately $10 \% 93$, though the clinical relevance of this finding is unclear given the proven cardiovascular benefits of statin therapy in patients with normal glucose homeostasis, glucose intolerance, and diabetes. In the CTT meta-analysis, every one mmol/L reduction in LDL-C by statin therapy resulted in a non-significant increase in hemorrhagic stroke (RR 1.12, 95\% Cl [0.93-1.35], $p=0.2$ ), but this was offset by a greater reduction in ischemic strokes for the same per unit change in LDL-C (RR 0.79, 95\% Cl [0.74-0.85], $p<0.0001$ )(Figure 1$)^{20}$. Observational reports have sporadically linked statins with cognitive decline, cataracts, and cancer, but these observations have been refuted in multiple large randomized controlled studies ${ }^{20,94-97}$.

Ezetimibe is generally well tolerated, and in IMPROVE-IT there were no differences between the study groups in any of the prespecified safety endpoints or rates of drug 
discontinuation ${ }^{56}$. Isolated reports of ezetimibe-associated upper respiratory tract infection, diarrhea, myalgias and arthralgias exist, but are rare and have not been seen in significant excess in any clinical studies ${ }^{98}$.

Bempedoic acid is a prodrug that is exclusively metabolized in the liver and therefore may avoid the muscle-specific adverse effects associated with statin therapies. In CLEAR Harmony, the incidence of adverse events did not differ between the bempedoic acid and placebo groups. However, the bempedoic acid group did have a higher rate of adverse events leading to discontinuation of the assigned drug (10.9\% vs $7.1 \%, p=0.005)$ and a higher incidence of gout ( $1.2 \%$ vs. $0.3 \%, p=0.03)^{42}$, likely explained by bempedoic acid byproducts competing for uric acid transporters in the kidney. Combination of the CLEAR Harmony and CLEAR Wisdom trials revealed that the risk of gout events was even higher in those with a prior history of gout (11.2\% bempedoic acid vs $1.7 \%$ placebo), providing caution for use in this specific subgroup of patients ${ }^{42,43}$. Bempedoic acid may affect other lipid parameters, and a meta-analysis of 10 randomized trials of bempedoic acid therapy has noted a $6 \%$ decrease in $\mathrm{HDL}-\mathrm{C}$ among those on treatment 99 . Additionally, the FDA labeling for bempedoic acid warns of possible increased risk of tendon rupture with the drug, as early clinical trial experience demonstrated tendon rupture occurred in $0.5 \%$ of 2,009 patients treated with bempedoic acid versus o\% of 999 patients treated with placebo.

PCSK9 inhibitors appear to have a promising safety profile. For the two PCSK9 inhibitors approved for clinical use by the FDA (evolocumab, alirocumab), treatment has not been shown to increase the rates of adverse events, serious adverse events, or adverse events leading to drug discontinuation. In both trials there was a higher rate of injection-site reactions, but the majority of these were mild and did not lead to drug discontinuation ${ }^{64,67}$. Prospective evaluation of cognition has revealed no effects on executive function, memory, or psychomotor speed between the PCSK9 inhibitor evolocumab and placebo ${ }^{100,102}$. A similar study with alirocumab is ongoing (NCT02957682), though no difference in neurocognitive event rates from ODYSSEY OUTCOMES has been noted to date.

More broadly, PCSK9 inhibitors lead to profound LDL-C lowering and provide insight on the safety of lowering LDL-C beyond targets endorsed by current guidelines ${ }^{65,101}$. An analysis detailing the rates of safety events by achieved LDL-C at four weeks in the FOURIER trial showed no excess of multiple prespecified safety outcomes including increases of hepatic transaminases, creatinine kinase, neurocognitive events, new onset diabetes, cataracts, malignancy, hemorrhagic stroke, or non-cardiovascular death in patients achieving an LDL-C $<0.5 \mathrm{mmol} / \mathrm{L}(20 \mathrm{mg} / \mathrm{dL})$ at 4 weeks compared to those with higher achieved LDL-C levels. Additionally, patients with ultra-low LDL-C concentrations less than $0.26 \mathrm{mmol} / \mathrm{L}(10 \mathrm{mg} / \mathrm{dL})$ had similar rates of adverse events when compared to patients in the reference group of $L D L-C \geq 2.6 \mathrm{mmol} / \mathrm{L}(100 \mathrm{mg} / \mathrm{dL})^{66}$.

\section{LDL-C treatment targets in secondary prevention have lowered over time}

Given the mounting evidence that pharmacologic LDL-C lowering provides relative risk reduction in major adverse cardiovascular events directly proportional to the magnitude of LDL-C lowering, and the increasing data showing safety at very low LDL-C ranges, it should not be surprising to find that LDL-C treatment targets continue to decrease over time (Figure 4).

In 1985, the National Heart, Lung, and Blood Institute (NHLBI) established the Adult Treatment Panel (ATP), a panel of experts under the National Cholesterol Education Program seeking to detect, evaluate, and treat high blood cholesterol and reduce ASCVD 


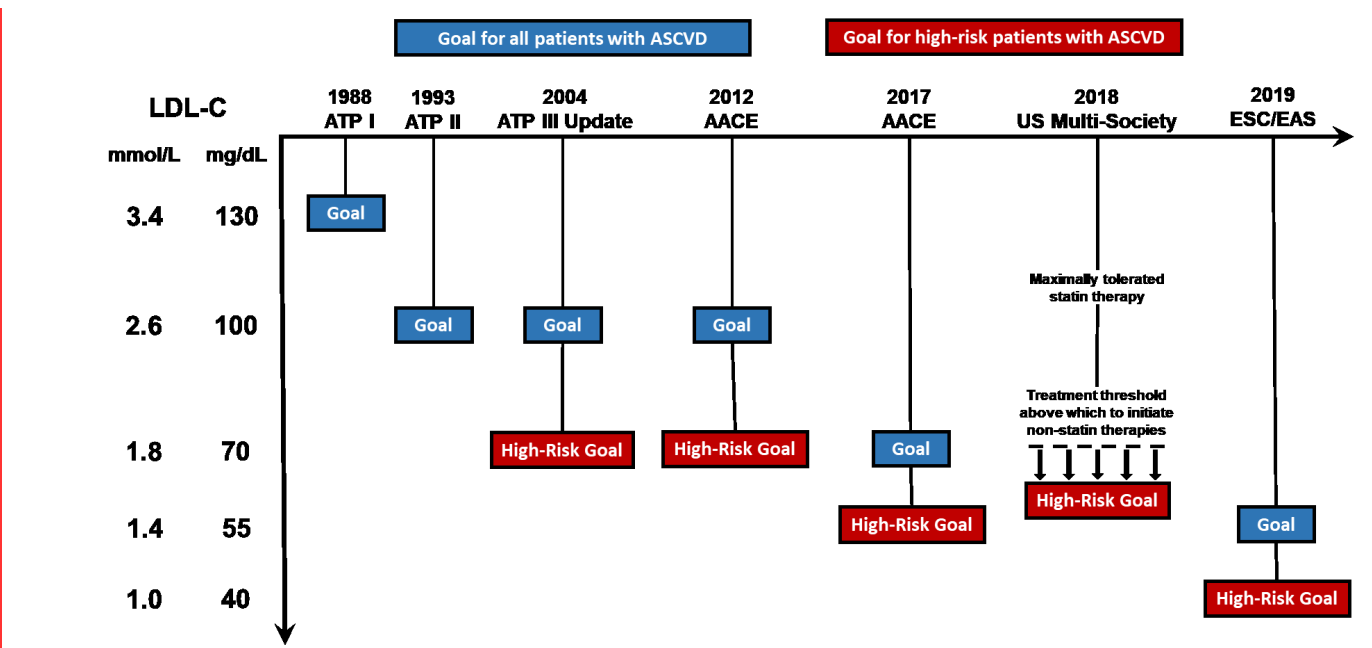

Figure 4. LDL-C treatment targets in cholesterol guidelines over time. LDL-C treatment targets were first suggested in 1988 by guidelines released by the Adult Treatment Panel and were broadly recommended for any individual at risk for ASCVD. In the three decades since, guidelines have suggested lower targets for those with established ASCVD and those who are at high risk for recurrent events. The most recent set of European guidelines from 2019 have recommended an LDL-C goal $<1.4 \mathrm{mmol} / \mathrm{L}$ ( 55 $\mathrm{mg} / \mathrm{dL}$ ) in high-risk populations, and a more aggressive target of $<1 \mathrm{mmol} / \mathrm{L}(40 \mathrm{mg} / \mathrm{dL})$ in patients experiencing recurrent events. ATP $=$ Adult Treatment Panel. AACE $=$ American Association of Clinical Endocrinologists. $\mathrm{ESC}=$ European Society of Cardiology. EAS $=$ European Atherosclerosis Society.

risk in adults. The first set of cholesterol guidelines, named ATP-I, was published in 1988 and outlined a strategy for primary prevention for coronary heart disease in individuals with elevated levels of $L D L-C^{103}$. The treatment strategy stated that a goal LDL-C of 3.4 $\mathrm{mmol} / \mathrm{L}(130 \mathrm{mg} / \mathrm{dL})$ in all individuals at risk for ASCVD was desirable. Notably, this first set of guidelines did not comment on secondary prevention in those with prior ASCVD events.

In the decades since, subsequent guidelines have incorporated targets for those with established ASCVD (ATP-II, 1993) ${ }^{104}$ and those deemed to be at very high risk for recurrent events (ATP-III, 2004) ${ }^{105}$. In the last five years, the American Association of Clinical Endocrinologists (AACE) and 2018 multi-society Blood Cholesterol Guidelines have supported the initiation of non-statin therapies to achieve LDL-C $<1.4 \mathrm{mmol} / \mathrm{L}$ $\left(55 \mathrm{mg} / \mathrm{dL}\right.$ ) for those at high risk ${ }^{10,106}$. Most recently, the ESC/EAS has recommended an LDL-C goal $<1.4 \mathrm{mmol} / \mathrm{L}(55 \mathrm{mg} / \mathrm{dL})$ for both primary and secondary prevention in high risk patients, with consideration of an even lower target of $<1 \mathrm{mmol} / \mathrm{L}(40 \mathrm{mg} / \mathrm{dL})$ for patients experiencing repeated events ${ }^{53}$.

\section{Key ongoing cardiovascular outcome trials}

Three important clinical outcomes trials are well underway that will add substantially to practice and may impact future guidelines (Figure 5).

CLEAR Outcomes (NCT02993406), the first phase 3 clinical outcomes trial studying bempedoic acid, has randomized 14,014 patients with statin intolerance, LDL-C $\geq 2.6$ $\mathrm{mmol} / \mathrm{L}(100 \mathrm{mg} / \mathrm{dL})$, and high risk for ASCVD to either bempedoic acid $180 \mathrm{mg}$ daily or matching placebo ${ }^{72}$. The primary endpoint is a composite of cardiovascular death, non-fatal MI, non-fatal stroke, or coronary revascularization and the estimated average treatment duration is 3.75 years. Results are anticipated in 2022-23.

VESALIUS-CV (NCT03872401), the next large trial of the PCSK9 inhibitor evolocumab, is designed to assess the efficacy of this therapy on the prevention of a first-ever 

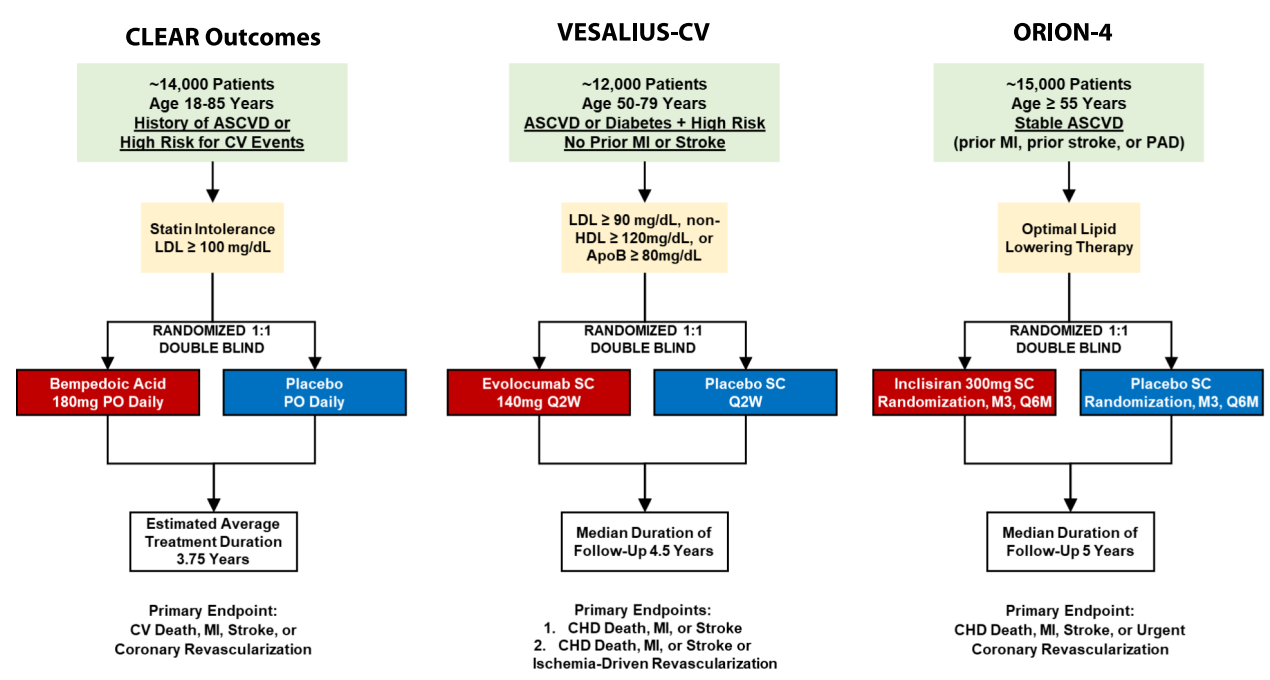

Figure 5. Key ongoing cardiovascular outcome trials of LDL-C lowering therapy. Three key trials are planned that are expected to inform clinical practice and impact future guidelines. CLEAR Outcomes (NCT02993406) will study the efficacy of bempedoic acid in reducing major cardiovascular events. VESALIUS-CV (NCT03872401) will assess the potential of PCSK9 inhibition in the prevention of a first-ever cardiovascular event. ORION-4 (NCT03705234) is investigating the clinical performance of inclisiran, an investigational cholesterol-lowering treatment.

cardiovascular event. Approximately 12,000 patients at high cardiovascular risk but no prior history of $\mathrm{Ml}$ or stroke will undergo 1:1 randomization to subcutaneous evolocumab or matching placebo. Two separate primary composite cardiovascular outcomes will be measured over a follow up of at least four years.

Lastly, ORION-4 (NCT03705234) is aiming to demonstrate safety and efficacy of inclisiran, a first-in-class small interfering RNA that inhibits PCSK9 synthesis and has recently been shown to reduce LDL-C levels by $50 \%$ in patients on maximally tolerated statin therapy ${ }^{107}$. Approximately 15,000 patients with prior ASCVD will be randomized to subcutaneous inclisiran sodium or matching placebo injections. Trial participants will be followed for the incidence of major adverse cardiovascular events over a median follow up period of five years. ORION-4 will provide important outcomes data for this latest investigational cholesterol-lowering treatment.

\section{CONCLUSION AND SUMMARY}

Patients with clinical ASCVD are at very high risk for future atherothrombotic events. Interventions directed at modifiable risk factors such as reducing levels of LDL-C are key features in the optimal management of these patients. Multiple lines of evidence suggest that decreases in plasma LDL-C particle concentration reduce the risk of major adverse cardiac events proportional to reduction in $\mathrm{LDL}-\mathrm{C}$ and the cumulative duration of exposure. For this reason, guidelines strongly support the use of LDL-C lowering therapy for secondary prevention in all patients.

Though lower LDL-C is strongly associated with lower risk for major adverse cardiac events, appropriate use of statins, ezetimibe, bempedoic acid, and PCSK9 inhibitors in at-risk populations is important for the most cost-effective utilization of these drugs. In general, all patients with a prior ASCVD event should begin treatment with maximally tolerated statin therapy. For high risk patients who remain above goal LDL-C levels on statin monotherapy, ezetimibe, bempedoic acid, and PCSK9 inhibitors should be strongly considered given data suggesting clinical benefit and no adverse safety outcomes from 
targeting LDL-C levels as low as $0.5 \mathrm{mmol} / \mathrm{L}(20 \mathrm{mg} / \mathrm{dL})$. Given the continued evidence supporting the "lower is better" hypothesis for goal LDL-C levels, it is expected that guidelines will continue to support lower LDL-C targets in the future.

\section{DISCLOSURES}

PNP has no disclosures. RPG has received grants from Amgen, Anthos Therapeutics, and Daiichi Sankyo; honoraria for CME lectures from Amgen, Daiichi Sankyo, and Merck; consultant fees from Amgen, American College of Cardiology, Astra Zeneca, BoehringerIngelheim, Bristol-Myers-Squibb, CryoLife, CVS Caremark, Daiichi Sankyo, Eli Lilly and Company, Esperion, Gilead, GlaxoSmithKline, Janssen, Lexicon, Merck, Pfizer, St. Lukes, SAJA Pharmaceuticals, Samsung, and Servier; and institutional research grants to the TIMI Study Group at Brigham and Women's Hospital for research he is not directly involved in from Abbott, Amgen, Aralez, AstraZeneca, Bayer HealthCare Pharmaceuticals, Inc., BRAHMS, Daiichi Sankyo, Eisai, GlaxoSmithKline, Intarcia, Janssen, Medlmmune, Merck, Novartis, Pfizer, Poxel, Quark Pharmaceuticals, Roche, Takeda, The Medicines Company, and Zora Biosciences.

\section{ACKNOWLEDGMENTS}

We would like to thank Christopher P. Cannon, M.D. for his independent and critical review of this manuscript.

\section{REFERENCES}

[1] Virani SS, et al. Heart disease and stroke statistics-2020 update: a report from the american heart association. Circulation. 2020;141(9):e139-e596.

[2] Roth GA, et al. Global, regional, and national burden of cardiovascular diseases for 10 causes, 1990 to 2015. J Am Coll Cardiol. 2017;70(1):1-25.

[3] Dagenais GR, et al. Variations in common diseases, hospital admissions, and deaths in middleaged adults in 21 countries from five continents (PURE): a prospective cohort study. Lancet. 2020;395(10226):785-794.

[4] Falk E. Pathogenesis of atherosclerosis. J Am Coll Cardiol. 2006;47(Suppl 8):C7-12.

[5] Libby P, et al. Atherosclerosis. Nat Rev Dis Primers. 2019;5(1):56.

[6] Ford ES, et al. Explaining the decrease in U.S. deaths from coronary disease, 1980-2000. N Engl I Med. 2007;356(23):2388-98.

[7] Emerging Risk Factors C. Lipid-related markers and cardiovascular disease prediction. JAMA. 2012;307(23):2499-506.

[8] Nordestgaard BG, et al. Familial hypercholesterolaemia is underdiagnosed and undertreated in the general population: guidance for clinicians to prevent coronary heart disease: consensus statement of the European Atherosclerosis Society. Eur Heart J. 2013;34(45):3478-90a.

[9] Navar-Boggan AM, et al. Hyperlipidemia in early adulthood increases long-term risk of coronary heart disease. Circulation. 2015;131(5):451-8.

[10] Grundy SM, et al. AHA/ACC/AACVPR/AAPA/ABC/ACPM/ADA/AGS/APhA/ASPC/NLA/PCNA Guideline on the Management of Blood Cholesterol: Executive Summary: A Report of the American College of Cardiology/American Heart Association Task Force on Clinical Practice Guidelines. J Am Coll Cardiol. 2018;73(24):3168-3209.

[11] Martin SS, et al. Comparison of a novel method vs the Friedewald equation for estimating low-density lipoprotein cholesterol levels from the standard lipid profile. JAMA. 2013;310(19):2061-2068.

[12] Miller WG, et al. Seven direct methods for measuring HDL and LDL cholesterol compared with ultracentrifugation reference measurement procedures. Clinical Chemistry. 2010;56(6):977-986.

[13] Nauck M, Warnick GR, Rifai N. Methods for measurement of LDL-cholesterol: a critical assessment of direct measurement by homogeneous assays versus calculation. Clin Chem. 2002;48(2):236-54.

[14] Doran B, et al. Prognostic value of fasting versus nonfasting low-density lipoprotein cholesterol levels on long-term mortality: insight from the National Health and Nutrition Examination Survey III (NHANESIII). Circulation. 2014;130(7):546-53.

[15] Miller M, et al. Triglycerides and cardiovascular disease: a scientific statement from the American Heart Association. Circulation. 2011;123(20):2292-333.

[16] Berglund L, et al. Evaluation and treatment of hypertriglyceridemia: an Endocrine Society clinical practice guideline. J Clin Endocrinol Metab. 2012;97(9):2969-89. 
[17] Musunuru K. Atherogenic dyslipidemia: cardiovascular risk and dietary intervention. Lipids. 2010;45(10):907-14.

[18] Castelli WP, et al. Incidence of coronary heart disease and lipoprotein cholesterol levels: the framingham study. JAMA. 1986;256(20):2835-2838.

[19] Sarwar N, et al. Triglycerides and the risk of coronary heart disease: 10,158 incident cases among 262, 525 participants in 29 Western prospective studies. Circulation. 2007;115(4):450-8.

[20] Cholesterol Treatment Trialists C, et al. Efficacy and safety of more intensive lowering of LDL cholesterol: a meta-analysis of data from 170, 000 participants in 26 randomised trials. Lancet. 2010;376(9753):1670-81.

[21] Group AS, et al. Effects of combination lipid therapy in type 2 diabetes mellitus. N Engl J Med. 2010;362(17):1563-74.

[22] Investigators A-H, et al. Niacin in patients with low HDL cholesterol levels receiving intensive statin therapy. N Engl J Med. 2011;365(24):2255-67.

[23] Group HTC. Effects of extended-release niacin with laropiprant in high-risk patients. N Engl J Med. 2014;371(3):203-12.

[24] Kaur N, et al. Effect of HDL-raising drugs on cardiovascular outcomes: a systematic review and metaregression. PLOS ONE. 2014;9(4):e94585.

[25] Lincoff AM, et al. Evacetrapib and Cardiovascular Outcomes in High-Risk Vascular Disease. N Engl J Med. 2017;376(20):1933-1942.

[26] Group HTRC, et al. Effects of anacetrapib in patients with atherosclerotic vascular disease. N Engl J Med. 2017;377(13):1217-1227.

[27] Armitage J, Holmes MV, Preiss D. Cholesteryl ester transfer protein inhibition for preventing cardiovascular events: JACC review topic of the week. J Am Coll Cardiol. 2019;73(4):477-487.

[28] Bhatt DL, et al. Cardiovascular risk reduction with icosapent ethyl for hypertriglyceridemia. N Engl J Med. 2019;380(1):11-22.

[29] Patel PN, Patel SM, Bhatt DL. Cardiovascular risk reduction with icosapent ethyl. Curr Opin Cardiol. 2019;34(6):721-727.

[30] Buhaescu I, Izzedine H. Mevalonate pathway: a review of clinical and therapeutical implications. Clin Biochem. 2007:40(9-10):575-84.

[31] Lennernas H, Fager G. Pharmacodynamics and pharmacokinetics of the HMG-CoA reductase inhibitors, Similarities and differences. Clin Pharmacokinet. 1997;32(5):403-25.

[32] Nicholls SJ, et al. Meta-analysis of comparative efficacy of increasing dose of Atorvastatin versus Rosuvastatin versus Simvastatin on lowering levels of atherogenic lipids (from VOYAGER). Am J Cardiol. 2010;105(1):69-76.

[33] Leitersdorf E. Cholesterol absorption inhibition: filling an unmet need in lipid-lowering management. European Heart Journal Supplements. 2001;3(suppl_E):E17-E23.

[34] Welder G, et al. High-dose atorvastatin causes a rapid sustained increase in human serum PCSK9 and disrupts its correlation with LDL cholesterol. J Lipid Res. 2010;51(9):2714-21.

[35] Vavlukis M, Vavlukis A. Adding ezetimibe to statin therapy: latest evidence and clinical implications. Drugs Context. 2018;7:212534.

[36] Huijgen R, et al. Colesevelam added to combination therapy with a statin and ezetimibe in patients with familial hypercholesterolemia: a 12-week, multicenter, randomized, double-blind, controlled trial. Clin Ther. 2010;32(4):615-25.

[37] Ross S, et al. Effect of bile acid sequestrants on the risk of cardiovascular events: a mendelian randomization analysis. Circ Cardiovasc Genet. 2015;8(4):618-27.

[38] Alder $\mathrm{M}$, et al. A meta-analysis assessing additional LDL-C reduction from addition of a bile acid sequestrant to statin therapy. Am J Med 2020;.

[39] Dadu RT, Ballantyne CM. Lipid lowering with PCSK9 inhibitors. Nat Rev Cardiol. 2014;11(10):563-75.

[40] Bergeron N, et al. Proprotein convertase subtilisin/kexin type 9 inhibition: a new therapeutic mechanism for reducing cardiovascular disease risk. Circulation. 2015;132(17):1648-66.

[41] Markham A. Bempedoic acid: first approval. Drugs. 2020;80(7):747-753.

[42] Ray KK, et al. Safety and efficacy of bempedoic acid to reduce LDL cholesterol. N Engl I Med. 2019;380(11):1022-1032.

[43] Goldberg AC, et al. Effect of bempedoic acid vs placebo added to maximally tolerated statins on lowdensity lipoprotein cholesterol in patients at high risk for cardiovascular disease: The CLEAR wisdom randomized clinical trial. JAMA. 2019;322(18):1780-1788.

[44] Laufs U, et al. Efficacy and safety of bempedoic acid in patients with hypercholesterolemia and statin intolerance. J Am Heart Assoc. 2019;8(7):e011662.

[45] Banach M, et al. Association of bempedoic acid administration with atherogenic lipid levels in phase 3 randomized clinical trials of patients with hypercholesterolemia. JAMA Cardiol 2020;.

[46] Ballantyne CM, et al. Bempedoic acid plus ezetimibe fixed-dose combination in patients with hypercholesterolemia and high CVD risk treated with maximally tolerated statin therapy. Eur J Prev Cardiol. 2020;27(6):593-603.

[47] Silverman MG, et al. Association between lowering LDL-C and cardiovascular risk reduction among different therapeutic interventions: a systematic review and meta-analysis. JAMA. 2016;316(12):1289-97.

[48] Ference BA, et al., Trialists Collaboration Reduction of low density lipoprotein-cholesterol and cardiovascular events with proprotein convertase subtilisin-kexin type 9 (PCSK9) inhibitors and statins: an analysis of FOURIER, SPIRE, and the Cholesterol Treatment. Eur Heart J. 2018;39(27):2540-2545. 
[49] Sabatine MS. PCSK9 inhibitors: clinical evidence and implementation. Nat Rev Cardiol. 2019;16(3):155-165.

[50] Ference BA. Mendelian randomization studies: using naturally randomized genetic data to fill evidence gaps. Curr Opin Lipidol. 2015;26(6):566-71.

[51] Ference BA, et al. Low-density lipoproteins cause atherosclerotic cardiovascular disease. 1. Evidence from genetic, epidemiologic, and clinical studies. A consensus statement from the European Atherosclerosis Society Consensus Panel. Eur Heart J. 2017;38(32):2459-2472.

[52] Collins R, et al. Interpretation of the evidence for the efficacy and safety of statin therapy. Lancet. 2016;388(10059):2532-2561.

[53] Mach F, et al. 2019 ESC/EAS Guidelines for the management of dyslipidaemias: lipid modification to reduce cardiovascular risk: The Task Force for the management of dyslipidaemias of the European Society of Cardiology (ESC) and European Atherosclerosis Society (EAS). European Heart Journal. 2019;41(1):111-188.

[54] Cholesterol Treatment Trialists C. Efficacy and safety of statin therapy in older people: a meta-analysis of individual participant data from 28 randomised controlled trials. Lancet. 2019;393(10170):407-415.

[55] Gencer B, et al. Efficacy and safety of lowering LDL cholesterol in older patients: a systematic review and meta-analysis of randomised controlled trials. The Lancet.

[56] Cannon CP, et al. Ezetimibe added to statin therapy after acute coronary syndromes. N Engl J Med. 2015;372(25):2387-97.

[57] Giugliano RP, et al. Long-term safety and efficacy of achieving very low levels of low-density lipoprotein cholesterol : a prespecified analysis of the IMPROVE-IT trial. JAMA Cardiology. 2017;2(5):547-555.

[58] Bohula EA, et al. Atherothrombotic risk stratification and ezetimibe for secondary prevention. J Am Coll Cardiol. 2017;69(8):911-921.

[59] Bach RG, et al. Effect of simvastatin-ezetimibe compared with simvastatin monotherapy after acute coronary syndrome among patients 75 years or older: a secondary analysis of a randomized clinical trial. JAMA Cardiology. 2019;4(9):846-854.

[6o] Bohula EA, et al. Prevention of stroke with the addition of ezetimibe to statin therapy in patients with acute coronary syndrome in IMPROVE-IT (Improved Reduction of Outcomes: Vytorin Efficacy International Trial). Circulation. 2017;136(25):2440-2450.

[61] Eisen A, et al. The benefit of adding ezetimibe to statin therapy in patients with prior coronary artery bypass graft surgery and acute coronary syndrome in the IMPROVE-IT trial. Eur Heart J. 2016;37(48):3576-3584.

[62] Giugliano RP, et al. Benefit of adding ezetimibe to statin therapy on cardiovascular outcomes and safety in patients with versus without diabetes mellitus: results from IMPROVE-IT (Improved Reduction of Outcomes: Vytorin Efficacy International Trial). Circulation. 2018;137(15):1571-1582.

[63] Bonaca MP, et al. Polyvascular disease, type 2 diabetes, and long-term vascular risk: a secondary analysis of the IMPROVE-IT trial. Lancet Diabetes Endocrinol. 2018;6(12):934-943.

[64] Sabatine MS, et al. Evolocumab and clinical outcomes in patients with cardiovascular disease. $N$ Engl J Med. 2017;376(18):1713-1722.

[65] Giugliano RP, et al. Clinical efficacy and safety of evolocumab in high-risk patients receiving a statin: secondary analysis of patients with low Idl cholesterol levels and in those already receiving a maximalpotency statin in a randomized clinical trial. JAMA Cardiol. 2017;2(12):1385-1391.

[66] Giugliano RP, et al. Clinical efficacy and safety of achieving very low LDL-cholesterol concentrations with the PCSK9 inhibitor evolocumab: a prespecified secondary analysis of the FOURIER trial. Lancet. 2017;390(10106):1962-1971.

[67] Schwartz GG, et al. Alirocumab and cardiovascular outcomes after acute coronary syndrome. N Engl] Med. 2018;379(22):2097-2107.

[68] Sabatine MS, Giugliano RP. Low-density lipoprotein cholesterol treatment in the proprotein convertase subtilisin/kexin type 9 inhibitor era: getting back on target. JAMA Cardiol. 2017;2(9):935-936.

[69] Koren MJ, et al. Long-term efficacy and safety of evolocumab in patients with hypercholesterolemia. J Am Coll Cardiol. 2019;74(17):2132-2146.

[70] Ballantyne CM, et al. Effect of ETC-1002 on serum low-density lipoprotein cholesterol in hypercholesterolemic patients receiving statin therapy. American Journal of Cardiology . 2016;117(12):1928-1933.

[71] Ballantyne CM, et al. Efficacy and safety of bempedoic acid added to ezetimibe in statin-intolerant patients with hypercholesterolemia: A randomized, placebo-controlled study. Atherosclerosis. 2018;277:195-203.

[72] Nicholls SJ, et al. Rationale and design of the CLEAR-outcomes trial: Evaluating the effect of Bempedoic acid on cardiovascular events in patients with statin intolerance. American Heart Journal 2020;.

[73] Thompson GR, Group H-ULAW. Recommendations for the use of LDL apheresis. Atherosclerosis. 2008;198(2):247-55.

[74] Bambauer R, et al. LDL-apheresis: technical and clinical aspects. ScientificWorldJournal. 2012;2012:314283.

[75] Hemphill LC. Familial hypercholesterolemia: current treatment options and patient selection for lowdensity lipoprotein apheresis. J Clin Lipidol. 2010;4(5):346-9.

[76] Julius U. Current role of lipoprotein apheresis in the treatment of high-risk patients. J Cardiovasc Dev Dis. 2018;5(2).

[77] Thompson GR, Miller JP, Breslow JL. Improved survival of patients with homozygous familial hypercholesterolaemia treated with plasma exchange. Br Med J (Clin Res Ed). 1985;291(6510):1671-3.

[78] Keller C. LDL-apheresis in homozygous LDL-receptor-defective familial hypercholesterolemia: the Munich experience. Atheroscler Suppl. 2009;10(5):21-6. 
[79] Thompson G, Parhofer KG. Current role of lipoprotein apheresis. Curr Atheroscler Rep. 2019;21(7):26.

[80] Feingold KR, Grunfeld C. Lipoprotein Apheresis, in Endotext. South Dartmouth (MA); 2000.

[81] Mabuchi $\mathrm{H}$, et al. Long-term efficacy of low-density lipoprotein apheresis on coronary heart disease in familial hypercholesterolemia. Hokuriku-FH-LDL-Apheresis Study Group. Am J Cardiol. 1998;82(12):1489-95.

[82] Emmrich U, Hohenstein B, Julius U. Actual situation of lipoprotein apheresis in Saxony in 2013. Atheroscler Suppl. 2015;18:215-25.

[83] Heigl F, et al. Efficacy, safety, and tolerability of long-term lipoprotein apheresis in patients with LDLor Lp(a) hyperlipoproteinemia: Findings gathered from more than 36 , ooo treatments at one center in Germany. Atheroscler Suppl. 2015;18:154-62.

[84] Schatz U, et al. Most significant reduction of cardiovascular events in patients undergoing lipoproteinapheresis due to raised Lp(a) levels - A multicenter observational study. Atheroscler Suppl. 2017:30:246-252.

[85] Moriarty PM, et al. Alirocumab in patients with heterozygous familial hypercholesterolaemia undergoing lipoprotein apheresis: the ODYSSEY ESCAPE trial. Eur Heart J. 2016;37(48):3588-3595.

[86] Armitage J. The safety of statins in clinical practice. Lancet. 2007;370(9601):1781-90.

[87] Macedo AF, et al. Unintended effects of statins from observational studies in the general population: systematic review and meta-analysis. BMC Med. 2014;12:51.

[88] Golomb BA, Evans MA. Statin adverse effects. American Journal of Cardiovascular Drugs. 2008;8(6):373-418.

[89] Zhang H, et al. Discontinuation of statins in routine care settings: a cohort study. Ann Intern Med. 2013;158(7):526-34.

[90] Hovingh GK, et al. Identification and management of patients with statin-associated symptoms in clinical practice: A clinician survey. Atherosclerosis. 2016;245:111-7.

[91] Newman CB, et al. Statin safety and associated adverse events: a scientific statement from the american heart association. Arterioscler Thromb Vasc Biol. 2019;39(2):e38-e81.

[92] Wood FA, et al. N-of-1 trial of a statin, placebo, or no treatment to assess side effects. N Engl / Med. 2020;383:2182-2184.

[93] Sattar N, et al. Statins and risk of incident diabetes: a collaborative meta-analysis of randomised statin trials. Lancet. 2010;375(9716):735-42.

[94] Pedersen TR, et al. Safety and tolerability of cholesterol lowering with simvastatin during 5 years in the scandinavian simvastatin survival study. Archives of Internal Medicine. 1996;156(18):2085-2092.

[95] anonymous. MRC/BHF Heart Protection Study of cholesterol lowering with simvastatin in 20, 536 highrisk individuals: a randomised placebo-controlled trial. Lancet. 2002;360(9326):7-22.

[96] Trompet S, et al. Pravastatin and cognitive function in the elderly. Results of the PROSPER study. J Neurol. 2010;257(1):85-90.

[97] Rojas-Fernandez CH, et al. An assessment by the statin cognitive safety task force: 2014 update. J Clin Lipidol. 2014;8(3 Suppl):S5-16.

[98] Lloyd-Jones DM, et al. 2016 ACC expert consensus decision pathway on the role of non-statin therapies for ldl-cholesterol lowering in the management of atherosclerotic cardiovascular disease risk: a report of the american college of cardiology task force on clinical expert consensus documents. Journal of the American College of Cardiology. 2016;68(1):92-125.

[99] Cicero AFG, et al. Efficacy and safety of bempedoic acid for the treatment of hypercholesterolemia: A systematic review and meta-analysis. PLoS Med. 2020;17(7):e1003121.

[100] Giugliano RP, et al. Cognitive function in a randomized trial of evolocumab. N Engl / Med. 2017;377(7):633-643.

[101] Schwartz GG, et al. Clinical efficacy and safety of alirocumab after acute coronary syndrome according to achieved level of low-density lipoprotein cholesterol: a propensity score-matched analysis of the ODYSSEY OUTCOMES trial. Circulation. 2021. doi: 10.1161/CIRCULATIONAHA.120.049447.

[102] Gencer B, et al. Cognition after lowering LDL-cholesterol with evolocumab. J Am Coll Cardiol. 2020;75(18):2283-2293.

[103] Goodman DS, et al. Report of the national cholesterol education program expert panel on detection, evaluation, and treatment of high blood cholesterol in adults. Archives of Internal Medicine. 1988;148(1):36-69.

[104] Grundy SM, et al. Summary of the Second Report of the National Cholesterol Education Program (NCEP) Expert Panel on Detection, Evaluation, and Treatment of High Blood Cholesterol in Adults (Adult Treatment Panel II). JAMA. 1993;269(23):3015-3023.

[105] Grundy SM, et al. Implications of recent clinical trials for the National Cholesterol Education Program Adult Treatment Panel III guidelines. Circulation. 2004;110(2):227-39.

[106] Jellinger PS, et al. American association of clinical endocrinologists and american college of endocrinology guidelines for management of dyslipidemia and prevention of cardiovascular disease. Endocr Pract. 2017;23(Suppl 2):1-87.

[107] Ray KK, et al. Two phase 3 trials of inclisiran in patients with elevated LDL Cholesterol. N Engl J Med. 2020;382(16):1507-1519. 